\title{
Comparison of ribitol and glycerol teichoic acid genes in Bacillus subtilis W23 and 168: identical function, similar divergent organization, but different regulation
}

\author{
Vladimir Lazarevic, François-Xavier Abellan,† Siham Beggah Möller, \\ Dimitri Karamata and Catherine Mauël
}

Author for correspondence: Catherine Mauël. Tel: +4121320 60 75. Fax: +41213206078. e-mail: catherine.mauel@igbm.unil.ch

Institut de Génétique et Biologie Microbiennes, Université de Lausanne, Rue César-Roux 19, $\mathrm{CH}-1005$ Lausanne, Switzerland
The tar genes directing the synthesis of poly(ribitol phosphate), the main teichoic acid in Bacillus subtilis strain W23, were sequenced. They are organized in two divergently transcribed operons, $\operatorname{tarABIJKL}$ and $\operatorname{tarDF}$, as are the tag genes specifying poly(glycerol phosphate) synthesis in B. subtilis 168. The features of the tar genes as well as the putative participation of their products in the proposed biosynthesis pathway of poly(ribitol phosphate) are presented. The tarA and tarD genes, which are most likely involved in the synthesis of the linkage unit (the entity coupling teichoic acid to peptidoglycan), are separated by $508 \mathrm{nt}$. Sequences of the outer segments of this regulatory region are similar to the two divergent promoter regions identified upstream of the tagA and tagD genes in strain 168. However, in W23, these regions, which also included functional promoters, are separated by an additional DNA segment of about $100 \mathrm{nt}$, on which two new mRNA starts, one in each direction, were identified. The regulatory regions of teichoic acid divergons of Bacillus globigii, Bacillus licheniformis and eight strains of $B$. subtilis were cloned and sequenced. In four $B$. subtilis strains and in B. globigii, their length and sequence are similar to the regulatory region of $W 23$. In the others, including B. licheniformis, they are of the 168-type. Analysis of nucleotide sequences of a non-coding grey hole, present in the tag region of strain 168, revealed higher similarities to tar than to tag entities. This suggests that at least part of the tag genes specifying the synthesis of glucosylated poly(glycerol phosphate) in strain 168 was introduced by horizontal gene transfer into a strain originally synthesizing a ribitol-phosphate-containing teichoic acid.

Keywords: poly(glycerol phosphate) origin, poly(ribitol phosphate) sequence, Bacillus evolution, anionic cell-wall polymers

\section{INTRODUCTION}

In Bacillus subtilis 168, five of the genes concerned with

† Present address: Biokema SA, Chemin de la Chatanerie 2, CH-1023 Crissier, Switzerland.

Abbreviations: -P, -phosphate; poly(GroP), poly(glycerol phosphate); poly(RboP), poly(ribitol phosphate); poly(GlcGalNAcP), poly(glucopyranosyl $\mathrm{N}$-acetylgalactosamine 1-phosphate).

The EMBL accession numbers for the nucleotide sequences reported in this paper are AJ313428, AJ318465, AJ318466, AJ318467, AJ318468, AJ318469 and AJ318470. the synthesis of the essential wall teichoic acid poly(glycerol phosphate) $[$ poly $(\mathrm{GroP})]$ are organized in a divergon, i.e. two divergently transcribed operons (tagAB and $\operatorname{tag} D E F)$, separated by a $399 \mathrm{nt}$ regulatory region (Mauël et al., 1991), in which two mRNA starts were mapped (Mauël et al., 1995). The tar region from $B$. subtilis W23 directs the synthesis of poly(ribitol phosphate) $[$ poly $(\mathrm{RboP})]$, a chemically different teichoic acid polymer (Armstrong et al., 1960). In both strains, the linkage unit which couples the teichoic acid main chain to peptidoglycan is formed by the same residues (Ward, 1981; Araki \& Ito, 1989). 
Table 1. Bacterial strains used in this study

\begin{tabular}{|c|c|c|}
\hline Strains & Genotype & Reference* \\
\hline \multicolumn{3}{|l|}{ Escherichia coli } \\
\hline $\mathrm{DH} 5 \alpha$ & $\begin{array}{l}\mathrm{F}^{-} \text {endA1 hsdR17 }\left(\mathrm{r}_{\mathrm{k}}^{-} \mathrm{m}_{\mathrm{k}}^{+}\right) \operatorname{supE44} \text { thi-1 recA1 } \\
\text { gyrA96(NalR) relA1 } \Delta(\operatorname{argF-lacZYA}) \mathrm{U} 169 \\
\phi 80 \text { dlacZ } \Delta \mathrm{M} 15 \text { deoR }\end{array}$ & Hanahan (1983) \\
\hline P2392 & $\begin{array}{l}\mathrm{F}^{-} \text {hsdR514 supE44 supF58 lacY1 galK2 galT22 } \\
\text { metB1 trpR55 }\left(\mathrm{P}^{+}\right)\end{array}$ & Genofit \\
\hline \multicolumn{3}{|l|}{ Bacillus strains } \\
\hline B. subtilis 168 & $\operatorname{trp} C 2$ & C. Anagnostopoulos \\
\hline B. subtilis W23 & Prototroph, $\mathrm{Sm}^{\mathrm{R}}$ & Lab. stock \\
\hline B. subtilis $\mathrm{S} 31$ & & Lab. stock \\
\hline B. subtilis $\mathrm{BS} 30$ & & T. Trautner \\
\hline B. subtilis BS41 & & T. Trautner \\
\hline B. subtilis BS121 & & T. Trautner \\
\hline B. subtilis BS129 & & T. Trautner \\
\hline B. subtilis BSG33-1 & & T. Trautner \\
\hline B. subtilis BSG40 & & T. Trautner \\
\hline B. subtilis BSG66 & & T. Trautner \\
\hline B. subtilis BSG150 & & T. Trautner \\
\hline B. subtilis var. natto & $\operatorname{amyE}(+N) \operatorname{amyR} 2 \operatorname{pro}(H)$ & BGSC 27A1 \\
\hline B. subtilis var. niger & $r p l V, \mathrm{Sm}^{\mathrm{R}}$ & BGSC 12A1 \\
\hline B. globigii & & BGSC 11A1 \\
\hline B. licheniformis & & BGSC 5A24 \\
\hline
\end{tabular}

*C. Anagnostopoulos, Laboratoire de Génétique Microbienne, INRA, France; T. Trautner, Max Planck Institute for Molecular Genetics, Berlin, Germany; BGSC, Bacillus Genetic Stock Center, Dept of Biochemistry, College of Biological Sciences, Ohio State University.

The expression of tag genes is subject to an elaborate control mechanism. First, there is evidence of an interdependence between teichoic acid and peptidoglycan synthesis (Ward, 1981). Second, under phosphate limitation, teichoic acid is replaced by teichuronic acid, a phosphate-free anionic polymer (Ellwood \& Tempest, 1969; Liu et al., 1998; Soldo et al., 1999). Transcriptional fusions to $\operatorname{tag} A$ and $\operatorname{tag} D$, the first genes of the $\operatorname{tag}$ operons (Mauël et al., 1994), confirmed that phosphate limitation was accompanied by reduced tag gene expression. In addition, these constructs revealed that, (i) under all experimental conditions used, $\operatorname{tag} A$ and $\operatorname{tag} D$ were apparently coordinately expressed, the level of $\operatorname{tag} D$ always being higher than that of $\operatorname{tag} A$; (ii) following the onset of sporulation, expression of tag genes diminished rapidly and was essentially abolished by stage II; and (iii) during germination, this activity was detectable before the rise in culture turbidity associated with spore outgrowth. Finally, the rate of $\operatorname{tag} A$ and $\operatorname{tag} B$ transcription increases at the end of the exponential phase of growth. Experiments in which transcription was monitored in media of different richness confirmed that higher growth rates were paralleled by lower transcription rates. This behaviour, characteristic of genes involved in septation (Vicente et al., 1991), suggested that tag gene transcription may also respond to cell-cycle-specific signals coupling chromosome replication and cell division (Mauël et al., 1995). Hybrids containing teichoic acid determinants specific to strain W23 in the 168 genetic background were constructed (Karamata et al., 1987). Southern hybridization analyses of nine such hybrids revealed that, in all of them, integral substitution of strain 168 tag genes by strain W23 tar genes had occurred (Young et al., 1989). Surface growth, flagellar motility, transformability and sporulation were normal (Karamata et al., 1987), suggesting that the signals regulating teichoic acid synthesis as a function of the environment, including hypothetical signals related to the cell cycle, were identical in the parent W23 and 168 strains. Therefore, sequence comparison of the regulatory region governing teichoic acid synthesis in these strains was expected to allow recognition of common cis-regulatory transcriptional signals.

In this contribution, we report the sequence and the genetic organization of the tar genes specifying the synthesis of poly (RboP) in B. subtilis W23, as well as the main features and presumed roles of their products. Their regulation appears more complex than that of the tag divergon in strain 168. The regulatory regions of the tar and tag divergons are analysed and compared to the equivalent region from ten other Bacillus strains. Finally, we present a new analysis of an untranslated 'grey hole' in the tag region (Lazarevic et al., 1995).

\section{METHODS}

Bacterial strains. These are listed in Table 1. 


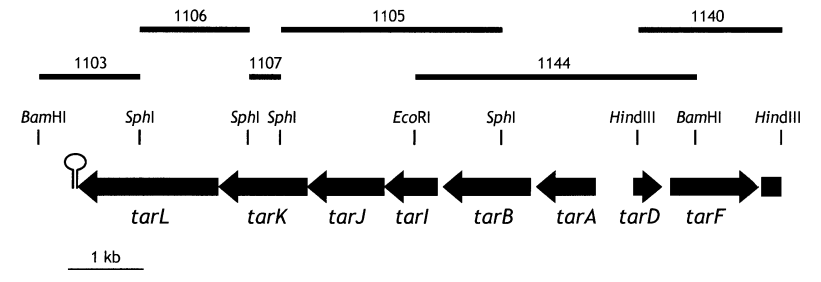

Fig. 1. Organization of genes involved in poly(RboP) synthesis in strain W23. Thick lines correspond to inserts in the named plasmid subclones. Relevant restriction sites are indicated. The stem-loop structure indicates a putative transcriptional terminator.

Cloning. A part of the $19 \mathrm{~kb}$ insert in the $\lambda 11$ clone, originating from the B. subtilis W23 chromosome (Young et al., 1989), was subcloned into the plasmid vector pMTL20EC (Chambers et al., 1988) (Fig. 1).

DNA sequencing and analysis. DNA sequencing was performed on both strands by primer-walking, using plasmid subclones of the $\lambda$ library. Labelling reactions were carried out with a Sequenase version $2.0 \mathrm{Kit}$ (USB) and $\left.{ }^{35} \mathrm{~S}\right] \mathrm{dATP} \alpha \mathrm{S}$ (Amersham). Junctions between certain subclones were verified by sequencing of $\lambda$ DNA. The sequence was compiled and analysed with the University of Wisconsin Genetic Computer Group software (Devereux et al., 1984).

DNA extraction and purification. Chromosomal DNA was extracted according to the method of Marmur (1961) and purified on $\mathrm{CsCl}$ density gradients in the presence of ethidium bromide (Sambrook et al., 1989). DNA bands were collected and dye-extracted with 1-butanol prior to precipitation (Sambrook et al., 1989). Plasmid DNA was prepared by the boiling method (Del Sal et al., 1988). $\lambda$ DNA was obtained as described by Grossberger (1987). Phage was propagated in Escherichia coli strain P2392.

PCR amplification. PCRs were set up with 10 ng chromosomal DNA, $200 \mathrm{pmol}$ of each primer and $20 \mathrm{nmol}$ of each dNTP (Biofinex) in $100 \mu \mathrm{l}$ reaction buffer containing 2.5 units AmpliTaq DNA polymerase (Perkin Elmer). Reactions were run with denaturation for $2 \mathrm{~min}$ at $95^{\circ} \mathrm{C}$, followed by 30 or 40 cycles of amplification $\left(95^{\circ} \mathrm{C}\right.$ for $1 \mathrm{~min}, 37^{\circ} \mathrm{C}$ for $1 \mathrm{~min}, 72^{\circ} \mathrm{C}$ for $1 \mathrm{~min}$ ) and final extension for $15 \mathrm{~min}$ at $72^{\circ} \mathrm{C}$. Teichoic acid regulatory regions were amplified with the following four oligonucleotide pairs: (i) 168-1D (5'-GCAGTAAATCAAAAGTTCCATATGTGATAACTTTTTTCAT-3') and 168-1A (5'-TGTTAAGTTACTGTTAACATAAGGAATATTGTGAATAGTC-3') ; (ii) 168-2D (5'-GCCTTCTGTTCTTGGGAGGTAAACAACCTC-3') and 168-2A (5'-CCTAAAGCTACGAATACCATGTCAGGATTTGCT-3'); (iii) W231D (5'-AGCTTCATATGTCCGTAGTGAAATAAATCAAATGTTCCGT-3') and W23-1A (5'-CTGTCTACAAAATCTAATTGATTAATGGGCTTTGTTTGC A-3'); (iv) W232D (5'-GCCATCTGTTCTTGGAAGGTAAATGACCTCGCA-3') and W23-2A (5'-CCTAACGCGCAAATACCATATCAGGAGTTGA-3'). Letters A and D refer to the genes in which the oligos were chosen, i.e. $\operatorname{tag} A / \operatorname{tar} A$ or $\operatorname{tag} D /$ tarD.

Cloning. PCR products were recovered from agarose gels (QIAquick gel extraction kit, Qiagen) and cloned in pUC18 (SureClone ligation kit, Pharmacia).

Transformation. E. coli DH5 $\alpha$ competent cells were prepared and transformed by the procedure of Chung \& Miller (1988).
Primer extension. RNA preparation and primer extension experiments were performed as previously described (Lazarevic et al., 1992) using 20 pmol of each of the W23-1D and W23-1A oligonucleotides (see above).

\section{RESULTS}

\section{Genes specifying the synthesis of poly(RboP) in W23 form a divergon}

A $\lambda$ clone encompassing the region specifying poly(RboP) synthesis in W23 (Young et al., 1989) was subcloned into plasmid vectors and sequenced. Analyses revealed eight ORFs organized in two putative divergently transcribed operons, $\operatorname{tar} A B I J K L$ and $\operatorname{tar} D F$ (Fig. 1). Their proximal genes, $\operatorname{tar} A$ and $\operatorname{tar} D$, are separated by $508 \mathrm{nt}$. The distal gene of the tarABIJKL operon is followed by a palindromic sequence that probably corresponds to a transcriptional terminator. The DNA segment extending between tarF and the downstream incomplete ORF, likely to be involved in the synthesis of the minor teichoic acid (Freymond, 1995), is apparently devoid of any transcriptional terminator signature. The main features of identified ORFs and their deduced products are summarized in Table 2.

Sequences of TarA, TarB, TarD and TarF proteins exhibit the highest similarity to their strain $168 \mathrm{Tag}$ counterparts, whose functions were, with the exception of TagA, identified biochemically (Pooley et al., 1991, 1992). Therefore, TarA, TarB and TarF most likely catalyse three successive steps in the synthesis of the teichoic acid linkage unit, i.e. the addition of $\mathrm{N}$-acetylmannosamine and two glycerol-P residues, respectively, to the lipid-carrier-bound $\mathrm{N}$-acetylglucosamine (Fig. 2). TarD probably supplies CDP-glycerol, the glycerol-P donor for TarB and TarF (Pooley et al., 1991). Although both TarF and TagF use CDP-glycerol as substrate and despite sharing greater than $60 \%$ identity, they do not carry out identical functions. Namely, in strain W23 TarF is likely to be responsible for the addition of the second glycerol-P which completes the linkage unit, whereas in strain $168 \mathrm{TagF}$ polymerizes the complete glycerol-P chain onto the first residue (Pooley et al., 1992). This difference may be correlated with the fact that TagF is almost twice the size of TarF and that only its C-terminal moiety is homologous to TarF.

Although weak, sequence similarities between TarJ and a number of polyol dehydrogenases (not shown) suggest that this protein is a ribitol-5-P dehydrogenase (ribulose5 -P reductase) responsible for the conversion of ribulose 5 -P to ribitol 5-P. Subsequently, as presented in the hypothetical poly (RboP) biosynthetic pathway (Fig. 2), ribitol-P is converted into CDP-ribitol by TarI, the ribitol-5-P cytidylyltransferase. Both TarK and TarL possibly use CDP-ribitol as substrate and achieve the addition of the first ribitol-P residue and the polymerization of the ribitol-P chain, respectively. These enzymes share $36 \%$ identity and are more similar to each other 
Table 2. Features of putative tar genes and their deduced products

\begin{tabular}{|c|c|c|c|c|c|c|c|c|}
\hline \multirow[t]{2}{*}{ Gene } & \multirow[t]{2}{*}{ Position } & \multirow{2}{*}{$\begin{array}{l}\text { Deduced product } \\
\text { (aa) }\end{array}$} & \multirow[t]{2}{*}{ Putative function } & \multicolumn{5}{|c|}{ Similar protein $(\mathrm{s}):$} \\
\hline & & & & $\begin{array}{l}\text { Identity } \\
(\%)\end{array}$ & $\begin{array}{l}\text { Match length } \\
\text { (aa) }\end{array}$ & ORF & Organism name & Reference \\
\hline \multirow[t]{2}{*}{$\operatorname{tar} L$} & $129 \leftarrow 1988$ & 619 & Ribitol-P transferase & 36 & 382 & $\operatorname{tar} K$ & B. subtilis W23 & This study \\
\hline & & & & 34 & 397 & $\operatorname{orf3}$ & H. influenzae & Follens et al. (1999) \\
\hline \multirow[t]{2}{*}{$\operatorname{tar} K$} & $2007 \leftarrow 3176$ & 389 & Ribitol-P transferase & 36 & 382 & $\operatorname{tar} L$ & B. subtilis W23 & This study \\
\hline & & & & 32 & 387 & orf 3 & H. influenzae & Follens et al. (1999) \\
\hline tarJ & $3180 \leftarrow 4205$ & 341 & Ribitol-5-P dehydrogenase & 60 & 339 & SA0246 & Staphylococcus aureus & Kuroda et al. (2001) \\
\hline tarI & $4202 \leftarrow 4915$ & 237 & Ribitol-5-P cytidylyltransferase & 37 & 232 & acs1 & H. influenzae & Follens et al. (1999) \\
\hline $\operatorname{tar} B$ & $5004 \leftarrow 6155$ & 383 & Glycerophosphotransferase & 49 & 378 & $\operatorname{tag} B$ & B. subtilis 168 & $\begin{array}{l}\text { Mauël et al. (1991); } \\
\text { Pooley et al. (1991) }\end{array}$ \\
\hline $\operatorname{tar} A$ & $6251 \leftarrow 7024$ & 257 & $\mathrm{~N}$-Acetylmannosamine transferase & 59 & 257 & $\operatorname{tag} A$ & B. subtilis 168 & Mauël et al. $(1991 ; 1994)$ \\
\hline $\operatorname{tar} D$ & $7533 \rightarrow 7922$ & 129 & Glycerol-3-P cytidylyltransferase & 76 & 129 & $\operatorname{tag} D$ & B. subtilis 168 & $\begin{array}{l}\text { Mauël et al. (1991); } \\
\text { Pooley et al. (1991) }\end{array}$ \\
\hline $\operatorname{tar} F$ & $8026 \rightarrow 9210$ & 394 & Glycerophosphotransferase & 63 & 388 & $\operatorname{tag} F$ & B. subtilis 168 & $\begin{array}{l}\text { Honeyman \& Stewart (1989); } \\
\text { Pooley et al. }(1992)\end{array}$ \\
\hline
\end{tabular}

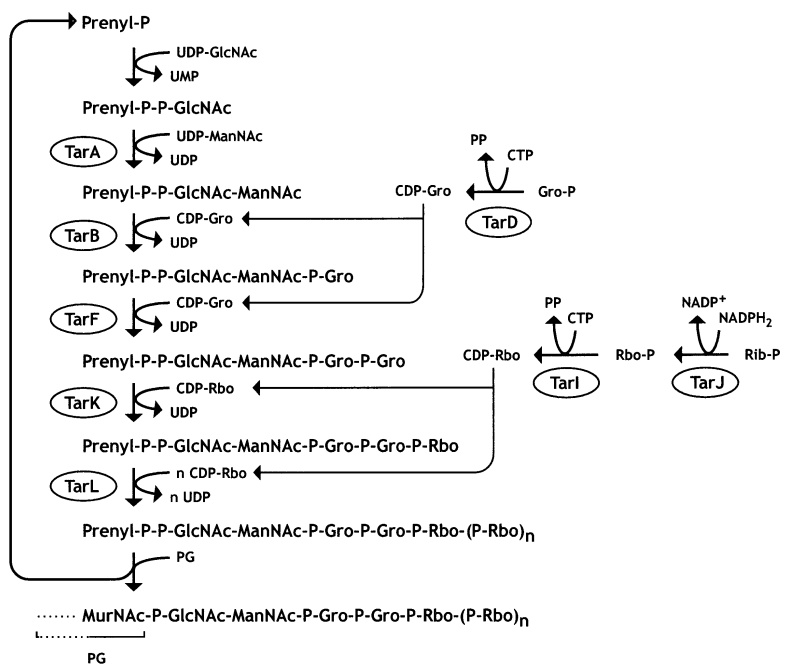

Fig. 2. Proposed participation of products encoded by the $\operatorname{tar} A B I J K L-\operatorname{tar} D F$ divergon in the biosynthesis of poly(RboP). Putative functions of tar gene products were deduced from sequence homologies. The biosynthesis pathway was adapted from Araki \& Ito (1989). GlcNAc, $N$-acetylglucosamine; Gro, glycerol; ManNAc, $\mathrm{N}$-acetylmannosamine; MurNAc, $\mathrm{N}$-acetylmuramic acid; PG, peptidoglycan; Rbo, ribitol; Rib, ribulose.

than to any other known protein. The observed similarity between TarK and TarL (Table 2) is restricted to the TarL C-terminal region, a situation analogous to the similarity between TagB and TagF. Again, the polymerization of the main chain apparently requires a much larger protein. In addition, TarK and the C-terminal moiety of TarL are similar, but to a lesser extent, to the product of orf3 (Follens et al., 1999) of Haemophilus influenzae. This Gram-negative bacterium synthesizes poly(ribosyl ribitol-P), a capsular polymer whose synthesis also requires the addition of ribitol-P.

\section{Sequencing of the regulatory region of the teichoic acid divergon from 10 strains of Bacillus: a DNA segment present in W23-like strains is absent from 168-like strains}

The regulatory regions of $\operatorname{tag} A B-\operatorname{tag} D E F$ and tarABIJKL-tarDF divergons consist of 399 and $508 \mathrm{nt}$, respectively. To explore the biological significance of this difference and to locate largely conserved sequences probably playing an important role in regulation, the teichoic acid regulatory region of a number of Bacillus strains was cloned and sequenced. Thirteen strains, including Bacillus globigii and Bacillus licheniformis, known, or predicted, to contain a linkage unit of either the 168 or the W23 type (Araki \& Ito, 1989), were screened by PCR for the presence of a $\operatorname{tag} A-\operatorname{tag} D$ or a $\operatorname{tar} A-\operatorname{tar} D$ regulatory region, with oligonucleotide pairs corresponding to the relevant genes (Table 3). For each strain, the PCR product, when obtained, was cloned and sequenced; six of them appeared to be of interest (see below and Table 3). The sequences of strains BS41, BS121 and BS129 were identical to that of strain 168, whereas the sequence of strain BSG66 did not differ from that of strain BSG150. B. subtilis var. natto, B. subtilis var. niger and strain BSG40 did not yield a PCR product, suggesting that the homologies with $\operatorname{tag} A D$ or $\operatorname{tar} A D$ are much lower, or that their teichoic acid genes are organized differently.

According to the size of their regulatory region, the six strains of interest fell into two groups (Table 3): those resembling W23, i.e. BSG150, B. subtilis S31 and B. globigii (although in the latter the length of the region is somewhat shorter), and those resembling 168, i.e. BSG33-1, BS30 and B. licheniformis. Sequence analyses confirmed this distribution. Strains carrying the longer regulatory regions, i.e. of a size close to that of strain W23, presented more extensive homologies to W23 than to strain 168 and vice versa (Fig. 3). The additional material, about $100 \mathrm{nt}$, present in W23 and W23-like strains was not dispersed within the 168-type sequences, 
Table 3. Size of teichoic acid regulatory regions of different Bacillus strains

Oligonucleotide pairs 168-1A/168-1D and W23-1A/W23-1D are immediately adjacent to the teichoic acid regulatory region of strains 168 and W23, respectively. Oligonucleotides 168-2A, 1682D, W23-2A and W23-2D correspond to highly conserved (over $80 \%$ identity) nucleotide sequences in $\operatorname{tag} / \operatorname{tar} A$ and $\operatorname{tag} / \operatorname{tar} D$.

\begin{tabular}{|c|c|c|c|c|c|}
\hline \multirow[t]{3}{*}{ Strain } & \multicolumn{4}{|c|}{ Oligonucleotide pairs* } & \multirow{3}{*}{$\begin{array}{l}\text { Regulatory region } \\
\text { (bp) }\end{array}$} \\
\hline & 168-1A & $168-2 \mathrm{~A}$ & W23-1A & W23-2A & \\
\hline & 168-1D & $168-2 \mathrm{D}$ & W23-1D & W23-2D & \\
\hline B. subtilis W23 & ND & ND & + & + & 508 \\
\hline B. subtilis BSG150 & - & ND & $+c$ & + & 508 \\
\hline B. subtilis S31 & - & ND & $+c$ & $\mathrm{ND}$ & 492 \\
\hline B. globigii & - & - & +- & $+c$ & 460 \\
\hline B. subtilis BSG33-1 & $+c$ & + & ND & ND & 408 \\
\hline B. subtilis $\mathrm{BS} 30$ & +- & $+c$ & - & - & 396 \\
\hline B. licheniformis & ND & $+c$ & - & - & 359 \\
\hline B. subtilis 168 & + & + & $\mathrm{ND}$ & $\mathrm{ND}$ & 399 \\
\hline
\end{tabular}

* + And -, presence and absence of PCR product, respectively. + - Indicates the presence of several low-intensity bands. c, Cloned. ND, Not done.

but represented a single segment located between the two divergent promoters identified in strain 168 (Mauël et al., 1995). The sequence of this segment was highly conserved in strains W23, BSG150 and S31, suggesting that it may play an important role in regulation (see below).

Similarities between the two groups were limited to the promoter regions identified in strain 168 . On the $\operatorname{tag} D$ side of the regulatory region, the entire $\mathrm{P}_{\text {tagD }}$ promoter region was highly similar to the corresponding sequence in W23-like strains. On the $\operatorname{tag} A$ side, a sequence identical to the $\mathrm{P}_{t a g A}-10$ region was identified in W23-like strains (TAAAAT). However, the highest similarities corresponded to strain 168 regions comprising motifs involved in the binding of PhoP and PhoP P (Liu et al., 1998). These proteins regulate the transition from teichoic to teichuronic acid synthesis as a response to phosphate limitation. In both groups, these binding sites were located in the promoter region, overlapping the probable -10 and -35 sequences in 168 -like strains and the -35 region in the other group (see below). Although these motifs may be located slightly differently relative to RNA-polymerase-binding sites, their presence suggests, nevertheless, that the teichoic/teichuronic switch is present in all of these organisms.

\section{The tar divergon is controlled by four promoters, two of which are located on the additional $100 \mathrm{nt}$ DNA segment}

To confirm that sequences homologous to the divergent promoters in strain 168 were functional in strain W23,
mRNA starts were mapped by primer-extension analyses with total RNA extracted from cells grown in LB medium, to $\mathrm{OD}_{600} 1.4$. Surprisingly, four promoters, two in each direction, were identified (Fig. 4). The outer ones, corresponding to those expected from sequence comparison with 168 , were designated $\mathrm{P}_{\text {tarA }}$-ext and $\mathrm{P}_{\text {tar } D}$-ext (ext, external with regard to the regulatory region). Two additional promoters, located in between, were designated $\mathrm{P}_{\text {tarA }}$-int and $\mathrm{P}_{\text {tarD }}$-int (int, internal with regard to the regulatory region). Their corresponding start sites were situated $107 \mathrm{nt}$ and 123 (127) nt upstream from the outer $\operatorname{tar} A$ and $\operatorname{tar} D$ mRNA starts, respectively.

The $\mathrm{P}_{\text {tarA }}$-int counterparts in the analysed W23-like strains probably correspond to active promoters, since their sequences are highly similar to that of the corresponding W23 segment. Their $\mathrm{P}_{t a r D}$-int counterparts are slightly less conserved, whereas $B$. globigii lacks about $20 \mathrm{nt}$ in this region.

\section{In strain 168 a $2 \cdot 2$ kb 'grey hole' contains remnants of tar genes from a W23-like strain}

The possibility of chromosomal rearrangements in strain 168 was already evoked when two non-coding segments of 0.7 and $2.2 \mathrm{~kb}$, designated 'grey holes', were found on each side of ggaAB, the operon specifying the synthesis of the so-called secondary, or minor teichoic acid, poly (glucopyranosyl $\mathrm{N}$-acetylgalactosamine 1-phosphate) [poly(GlcGalNAcP)] (Freymond, 1995; Lazarevic et al., 1995). Analyses revealed that these, apparently untranslated, regions comprised segments similar to neighbouring teichoic acid determinants. The 

CATCGTaTCAACCCTCCTTTTCATCTATATgTCTa A.AaAaaacacgTGTCATTCGTatAGTTTgatTAAAGCCtCTTtGcCIEA. AAACAET CATCgTaTCAACCCTCCTTTTCATCTATATgTCTatAaAaaatacgTGTCATTCgTatAGTTTGatTTAAAGaCtCTTtGtCTGA. AAACALT TATCaTaTCAACCCTCCTTTTCATCTATATaTCTCCAtA...... TGTaATCgT. .AGTTTagaTAAAGtCCCTTaGCCTGAtAAAgACT CATCaTgTCAACCCTaCTTTTCATCTATATgTCTatAaAaaa.acgTGTCATTCaTatAGTTTgatTAAAGaCtCTTtGCCTGA. AAACAtT

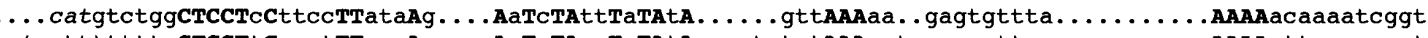
catagtttttt_cCTCCTCCgatTTaagA.....A.TgTAagTaTAtAcgaatgtatAAAagtcgaagattaagagaccgacccAAAAattcaggaggt .... catcaactcCTCCTсCcgatTTaaagttcaAaTtTAtgTtTAaAcgaaatga.AAAtgtcttaggaaagtggttcagcccAAAAatcccatcggt

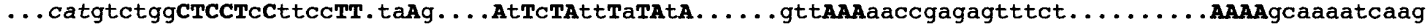
$\leftarrow \operatorname{tag} A$

TtACTC $t$ AtCTTACCACaAAAAtCTTTAGGAACTt. ttaacatt.CacATTACAATTCCTTCACgTTTTGACGATGAAtTAATATGAGGgaggGCGTaCA TtACTctAtCTTACCACaAAAATCTTTAGGAACTt. ttaacagttCacATTACAATTCCTTCACgTTTTGACGATGAATTAATATGAGG. aggGCGTaCA

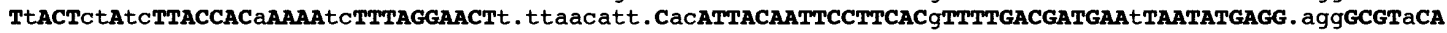
TCACTaaAt TTACCACgAAAAt TTTAGGAACTa........... gtATTACAATTCCTTCACgTTTTGACGATGAAgTAATATGAGG. aggGCGTaCA TtACTC $t$ ACCTTACCACaAAAAgCTTTAGGAACTt. t taacatt. CacATTACAATTCCTTCACCTTTTGACGATGAATAATATGAGG. . . aGCGTgCA $===============================$

CTAaCtTATATTTTAaCATAaATCtTCaACAAAgGCTTAACATT . ATCTTTACA t

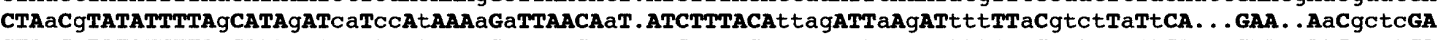
CTAaCgTATATTMPAaCATAgATgaTtCAtAAAaGCTTAACAaT. AACTTTACAacagATTaAgATtt tTTaCgCtgTCTtCA. . . GAA. . AtCagatGA

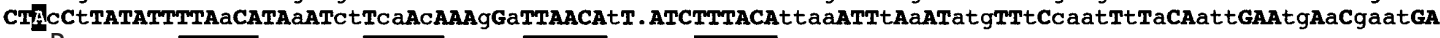
$\leftarrow \mathrm{P}_{\operatorname{tag} A}$ $\leftarrow \mathrm{P}_{\operatorname{tar} A^{-i n t}}$

$$
P_{\text {tarD }} \text {-int } \rightarrow
$$

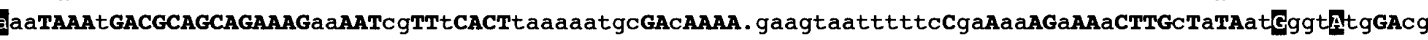
aaTAAATGACGCAGCAGAAAGaAAATC $T$ TtCACT $t a a a a a c g C G A C A A A A . ~ g a t t c g a t t t t t t C g a A a a A G a A A a C T T G t T a T A a t G g g t a t g G A t a$ gaaTAAATGACGCAGCAGAAAGaaAATC gTTtCACTtaaaaatgCGACAAAA. gaatcgatttttcCgaAaaAGaAAaCTTGtTaTAatGggtatgGAtg aaTAAATGACGCAGCAGAAAGaaAATCaTTaCACTtgaaaacgCGACAAAAagaattgactcttcCggAatAGaAAaCTTGtTgTAatGagtataGAta atgTAAAGGCGCAGCAGAAAGggAATtgTTtCACTgg . . . ctGAtAAA. . . . . . . aCaatcAGtAAgCTTGgTgTA. . GgaCgagGAtC

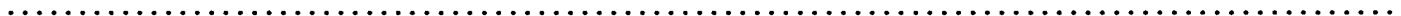

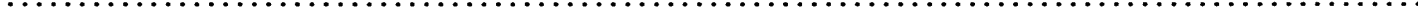

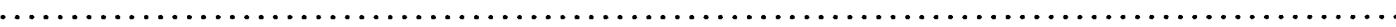

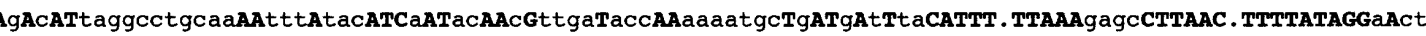

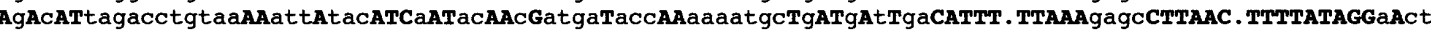
AgAcATtggacc tataaAAtt ta tacATCaATacAAcGgtgaTaccAaaaa $t g c T$ gATgAtTgaCATTT. TTAAAgagCCTTAAC. TTMTATAGGaAct AgAtAcagatctgtaaAAaatACacATCaATgCAACGatatTgtcAAaaagtgCTgAT gAtTtaCATTT. TTAAAgagCCTTAAC. TTTTATAGGaACa

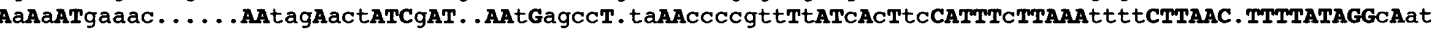
........ ataacaatccttgatgttaagggagttttatTggagttacAtATTTGgAaTgttTrtctccgcgtgAgCaCTTAACCTTTttTAGGCgg .

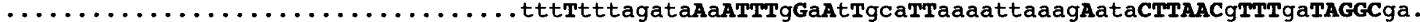

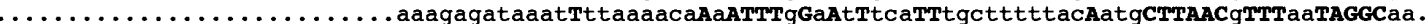
........ acaacagaccttggtgccaatggagtctgagTgtaattacAtATTTtGgAaTgttTTtctcctcatgAgtaCTTAACCTTTt tTAGGCgg.

$$
P_{\text {tarD }} \text {-ext } \rightarrow
$$

TATGCTATTATTMTCAtTATTAATGgtaCgTaAaAaaTatGTcGaAATATaAAcgatctGTtAAggtTTtTgGTtTTtT.tctggtactctcagTtga TATGCTATTATTGTTCAaTATTAATGgtaCgTaAaAaaTgtGTCGaAATtATaAAcaatc tGT tAAggtTTgTgGTtTTtT. tctggtactctcagTtga TATGCTATTATTGTCA TTATTAATGgtaCgTaAaAaaTacGTCGaAATATaAAcaatctGT tAAggtTTtTgGTtTTcTgtctacaaaatctaaTtga TATGCTATTATTGTTtAtTATTAATGgaaCgTaAaAaaTatGTCGaAATgATaAAcaatcaGTtAAggaTTtTtGTtTTtT.......... . taagTtgg TATGCTATTATTGTTaAaTATTAATGatgCaTgAgAttTatGTaGgAATtATtAAgagattGTCAAaaaTTtTgGTCTTtTCtctcaacc...... cTgaa $=========$

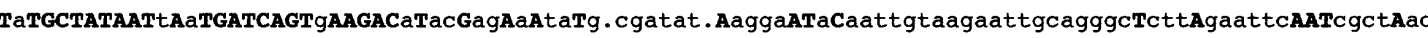

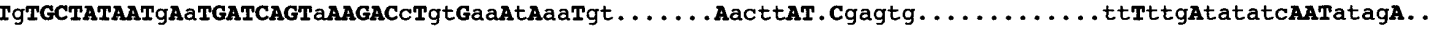

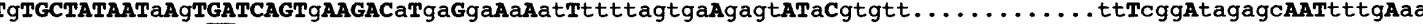
TaTGCTATAATt AaTGATCAGTgAAGACaTacGagAaAtaTgtcgatat. AaggaATaCaaa...........caggttT.ttAgaatttAATcgctAac $P_{\text {tag } D} \rightarrow$

aacggttggaaggtgatgttatctaatg aacggttggaaggtgatgttatctgatg ttaatgggctttg.............. aaaggttggaaggtgatgttatctaatg aggagttttaatgtg.............

gacagt.agtgttttTaaTaGtgTTtaAcATTAGgAAGGAGCGttacTttaaATG ........... TttTcGCCTTa. AgATTAGaAAGGAGaGca. . TgacaATG gttaatcatagtggtTtgTgGagTTatAgATTAGgAAGGAGaGtaatTtaacATG gtcagt.agtgttttTaaTaGtgTTtaACATTAGgAAGGAGcGtttcTttaaATG

Fig. 3. Nucleotide sequences of the regulatory regions controlling teichoic acid gene expression in eight strains of Bacillus, as well as of the relevant region in a 168 grey hole (see text). Only the $5^{\prime}$ to $3^{\prime}$ strands corresponding to the sense of chromosome replication in strain 168 are shown. Sequences are clustered into two groups. Conserved residues within each group are in uppercase. Putative start codons and their grey hole equivalent are in italic. Regions of high similarity between the two groups of sequences are indicated by $=$. Transcriptional starts in W23 and 168 are shaded black. Arrows indicate the direction of transcription/translation. Underlined sequences indicate motifs involved in PhoP-P binding (Liu et al., 1998). W23, 150 and S31, B. subtilis W23, 150 and S31, respectively; Bgl, B. globigii; grh, strain 168 relevant grey-hole region (Lazarevic et al., 1995); 168, 331 and BS30, B. subtilis 168, 331 and 30, respectively; Bli, B. licheniformis. 


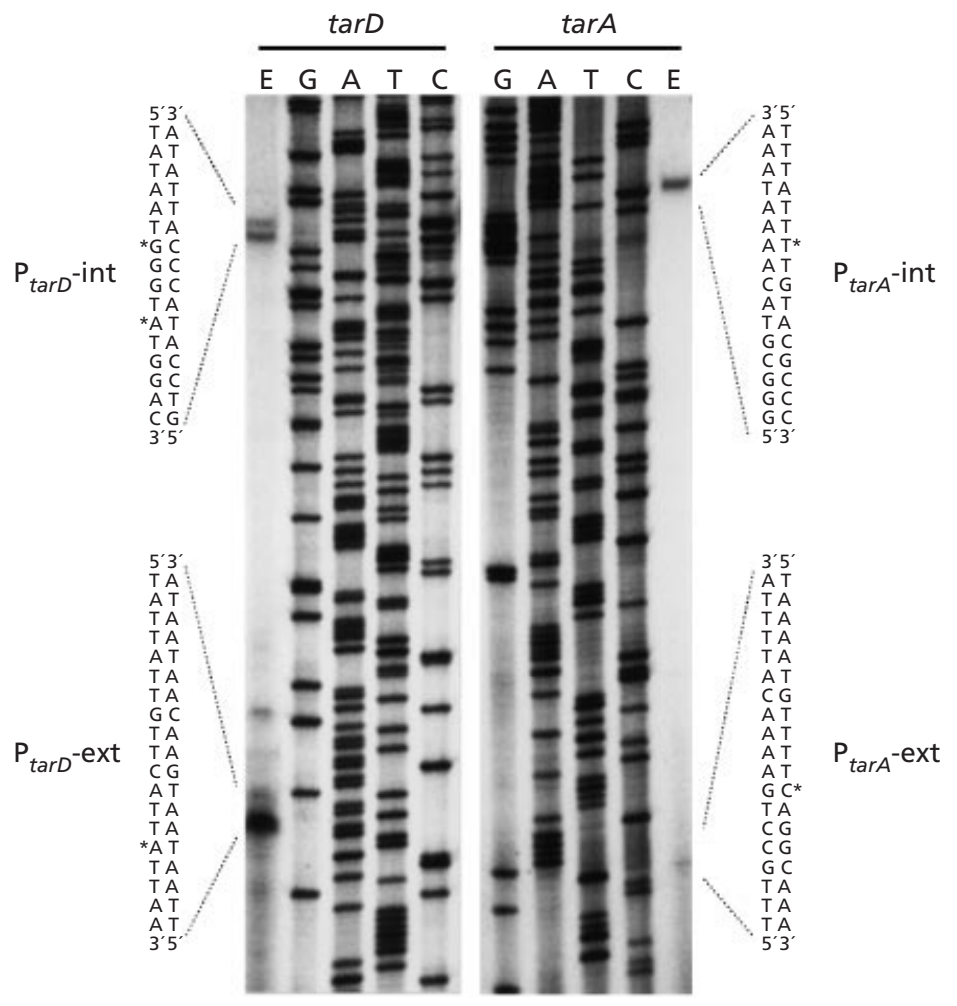

\begin{abstract}
Fig. 4. Determination, by extension of primers, of the 5 -ends of mRNA transcripts starting in the regulatory region of the strain W23 tar divergon. The products of primer extension reactions and sequencing reactions were generated with the same oligonucleotides, i.e. W23-1D and W23-1A on the $\operatorname{tar} D$ and $\operatorname{tar} A$ side, respectively. The nucleotide sequence surrounding each start (*) is indicated. G, A, T and C, sequence; E, extension.
\end{abstract}

nucleotide sequence of strain W23 teichoic acid genes sheds new light on the $2 \cdot 2 \mathrm{~kb}$ grey hole which apparently consists of three contiguous domains. One of them, $0.64 \mathrm{~kb}$ long, was reported to be $94 \%$ identical to the gtaB gene (Lazarevic et al., 1995). It is followed by a $0.75 \mathrm{~kb}$ segment that is $89 \%$ identical to the W23 region comprising the equivalent of the tar, not the tag, regulatory region and apparent remnants of the tarD gene (Fig. 5). The remaining $0.7 \mathrm{~kb}$ of the grey hole presents 65 and $62 \%$ identity to $\operatorname{tar} F$ and $\operatorname{tag} F$ genes, respectively. Remnants of tarD-like and tarF/tagF-like genes have obviously suffered large deletions amounting to 195 and $463 \mathrm{nt}$, respectively.

\section{DISCUSSION}

The experiments presented here have revealed that the synthesis of poly(RboP), the major teichoic acid of $B$. subtilis W23, is determined by a region comprising eight genes organized in divergently transcribed operons, tarABIJKL and tarDF. This contrasts with the situation in strain 168 , where the synthesis of poly $(\mathrm{GroP})$ requires only four genes. The latter are similarly organized, forming the $\operatorname{tag} A B-\operatorname{tag} D(E) F$ divergon, which contains a non-essential gene, tarE, responsible for the glucosylation of the polymer (Mauël et al., 1991). tarA and $\operatorname{tar} D$ genes, as well as their 168 counterparts $\operatorname{tag} A$ and $\operatorname{tag} D$, are located on each side of the regulatory region. They are involved in the synthesis of the linkage unit, an entity which couples the main teichoic acid chain to peptidoglycan and which is formed by the same residues in both strains. Sequences of TarA, TarB and TarF, which most likely catalyse three successive steps in the synthesis of the linkage unit, and the sequence of TarD, which probably synthesizes the CDP-glycerol precursor from glycerol-P, exhibit high similarity to their Tag counterparts. In strain 168 , the linkage unit comprises $\mathrm{N}$-acetylglucosamine, $\mathrm{N}$-acetylmannosamine and one glycerol-P residue onto which TagF polymerizes the main glycerol-P chain. However, the role of TarF, the TagF counterpart in W23, is possibly limited to the addition of a second glycerol-P residue to the 168-type linkage unit. To complete the ribitol-P polymer, four more hypothetical steps, i.e. four genes, are needed. These are the two steps required for the synthesis of CDP-ribitol from ribulose-P by successive action of TarJ and Tarl, the addition of a ribitol-P residue onto the second glycerol-P of the linkage unit by TarK and, finally, TarL-mediated polymerization of the ribitol-P chain. These four genes, specific to poly $(\mathrm{RboP})$ synthesis, are located downstream of $\operatorname{tar} A B$.

Expression of tar genes identified so far is regulated by four promoters, two in each direction, whereas the regulatory region of the tag divergon contains only two promoters (Mauël et al., 1995). The additional promoters in W23 are located on a DNA segment of about $100 \mathrm{nt}$, situated between homologues of the two 168 promoters. Sequence analysis of the teichoic acid regulatory regions of $B$. globigii, B. licheniformis and eight strains of $B$. subtilis revealed a clear-cut difference between W23- and 168-type strains. The latter, which 


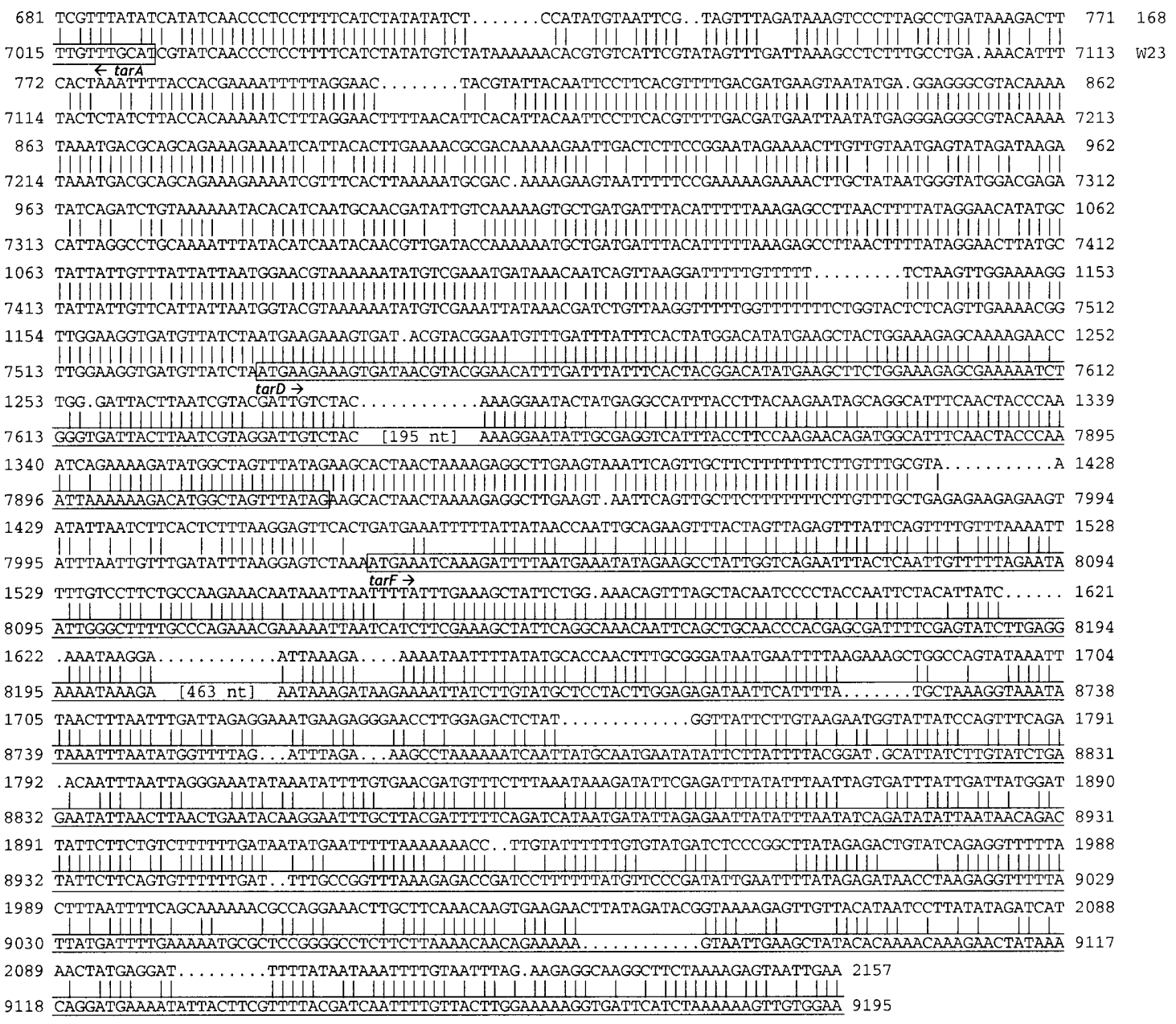

Fig. 5. Alignment of the grey-hole region of strain 168 with the similar sequences in strain $W 23 . \operatorname{tar} A, \operatorname{tar} B \operatorname{and} \operatorname{tar} D$ genes are boxed. Numbers of nucleotides forming the two long extra segments in W23 are indicated in parentheses. The GenBank accession numbers of sequences used in this alignment were AJ313428 (W23, this study) and U11099 (168, Lazarevic et al., 1995).

include B. licheniformis, were devoid of the central part of the regulatory region present in W23-like strains and corresponding to the two internal promoters of strain W23. So far, available evidence would suggest that this difference, i.e. the absence of the internal promoters, may be correlated with the nature of the synthesized anionic polymer. On the one hand, walls of S31, a W23type strain, contain poly $(\mathrm{RboP})$. On the other hand, although the nature of the anionic polymers of $B$. licheniformis BGSC 5A24 is not known, it was reported that the cell wall of B. licheniformis ATCC 9945, as well as that of strain AHU 1371, contain poly (GroP) (Chin et al., 1968; Araki \& Ito, 1989). B. globigii appears as an intermediate, since its teichoic acid regulatory region resembles that of W23, from which, however, part of the segment corresponding to $\mathrm{P}_{\operatorname{tarD}}$-int is missing, while its susceptibility to $B$. subtilis defective phages, i.e. PBSX resistant, PBSY and PBSZ sensitive (Mauël, unpublished), is identical to that of strain 168 (Karamata et al., 1987), suggesting that its wall polymer may be poly(GroP).
In the gene region for the teichoic acids of strain 168 , two non-coding grey holes, of 0.7 and $2.2 \mathrm{~kb}$, were found on each side of ggaAB, the operon which specifies the synthesis of poly(GlcGalNAcP), the minor teichoic acid. They comprise remnants of genes involved in the synthesis of the major teichoic acid and possibly reflect chromosome rearrangements generated by horizontal acquisition of the ggaAB operon (Lazarevic et al., 1995). Sequence determination of the tar divergon sheds new light on the $2 \cdot 2 \mathrm{~kb}$ grey hole, apparently the longest non-coding region of the $B$. subtilis 168 chromosome (Kunst et al., 1997). Indeed, at variance with a previous conclusion, a $1.5 \mathrm{~kb}$ segment of this grey hole bears more resemblance to the tar regulatory region as well as to tar, rather than tag, genes. These observations suggest that, like the ggaA operon, at least some of the genes encoding poly $(\mathrm{GroP})$ synthesis in strain 168 were acquired by horizontal transfer (Lazarevic et al., 1995), possibly displacing all or part of the resident W23-like polyribitol-synthesizing genes. This event could have generated the observed chromosome rearrangements and 
deletions. It is also possible that the acquisition of a cassette allowing polymerization of glycerol-P residues, originally designed for the synthesis of the linkage unit only, represented a distinct selective advantage (e.g. the economy realized by the substitution of a 5 -carbon for a 3 -carbon molecule) which might have culminated in the replacement of poly $(\mathrm{RboP})$ by poly $(\mathrm{GroP})$ synthesis, and the elimination of tarIJKL genes. A more extensive comparison between the complete tag and tar regions of 168 and W23, as well as their comparison to equivalent regions of other Bacillus strains, in particular strains containing poly(GroP) in their wall, may yield new elements and possibly allow a partial reconstitution of the events which, in strain 168 , led to the disturbed genetic organization of the teichoic acid locus and its interruption by two grey holes (Lazarevic et al., 1995).

The major teichoic acids in strains 168 and W23 perform analogous functions. Not only do conditional lethal mutants affected in their synthesis exhibit the same phenotype, but also exchange of the 168 poly (GroP) for the poly(RboP) entity (Karamata et al., 1987) does not entail any obvious phenotypic alteration. In these hybrids, the synthesis of the major anionic polymer apparently goes hand in hand with that of peptidoglycan, decreasing under phosphate limitation, while apparently remaining closely related to the cell cycle. However, the regulation of the tar and tag divergons is different. As revealed by primer-extension mapping, the synthesis of poly $(\mathrm{RboP})$ requires four promoters, whereas only two are needed for the synthesis of poly $(\mathrm{GroP})$. In our opinion, these observations raise an interesting question, i.e. what is the strategy used by 168 to apparently achieve, with two promoters, the same, finely tuned, regulation as W23, endowed with four functional promoters? Investigating the role of the internal promoters in the latter strain should help in recognizing the regulatory devices elaborated by strain 168 to palliate their absence in the tag regulatory region.

\section{ACKNOWLEDGEMENTS}

We thank Armelle Bauduret for excellent technical assistance. The financial support of the Fonds National Suisse de la Recherche Scientifique (grants 31-32588.91 and 31-45910.95) is also gratefully acknowledged.

\section{REFERENCES}

Araki, Y. \& Ito, E. (1989). Linkage units in cell walls of Grampositive bacteria. Crit Rev Microbiol 17, 121-135.

Armstrong, J. J., Baddiley, J. \& Buchanan, J. G. (1960). Structure of the ribitol teichoic acid from walls of Bacillus subtilis. Biochem J 76, 610-621.

Chambers, S. P., Prior, S. E., Barstow, D. A. \& Minton, N. P. (1988). The PMTL nic-cloning vector. I. Improved pUC polylinker regions to facilitate the use of sonicated DNA for nucleotide sequencing. Gene 68, 139-149.

Chin, T., Younger, J. \& Glaser, L. (1968). Synthesis of teichoic acid.
VII. Synthesis of teichoic acids during spore germination. J Bacteriol 95, 2024-2050.

Chung, C. T. \& Miller, R. H. (1988). A rapid and convenient method for the preparation and storage of competent bacterial cells. Nucleic Acids Res 16, 3580.

Del Sal, G., Manfioletti, G. \& Schneider, C. (1988). A one-tube plasmid DNA mini-preparation suitable for sequencing. Nucleic Acids Res 16, 9878.

Devereux, J., Haeberli, P. \& Smithies, O. (1984). A comprehensive set of sequence analysis programs for the VAX. Nucleic Acids Res 12, 387-395.

Ellwood, D. C. \& Tempest, D. W. (1969). Control of teichoic acid and teichuronic acid biosynthesis in chemostat cultures of Bacillus subtilis var. niger. Biochem J 111, 1-5.

Follens, A., Veiga-da-Cunha, M., Merckx, R., van Schaftingen, E. \& van Eldere, J. (1999). acs1 of Haemophilus influenzae type a capsulation locus region II encodes a bifunctional ribulose 5phosphate reductase-CDP-ribitol pyrophosphorylase. J Bacteriol 181, 2001-2007.

Freymond, P.-P. (1995). Génétique et biochimie des acides téichoïques secondaires de Bacillus subtilis 168 et W23. PhD thesis, Lausanne University, Switzerland.

Grossberger, D. (1987). Minipreps of DNA from bacteriophage lambda. Nucleic Acids Res 15, 6737.

Hanahan, D. (1983). Studies on transformation of Escherichia coli with plasmids. J Mol Biol 166, 557-580.

Honeyman, A. L. \& Stewart, G. C. (1989). The nucleotide sequence of the rodC operon of Bacillus subtilis. Mol Microbiol 3, 1257-1268.

Karamata, D., Pooley, H. M. \& Monod, M. (1987). Expression of heterologous genes for wall teichoic acids in Bacillus subtilis 168. Mol Gen Genet 207, 73-81.

Kunst, F., Ogasawara, N., Moszer, I. \& 148 other authors (1997). The complete genome sequence of the Gram positive bacterium Bacillus subtilis. Nature 390, 249-256.

Kuroda, M., Ohta, T., Uchiyama, I. \& 34 other authors (2001). Whole genome sequencing of methicillin-resistant Staphylococcus aureus. Lancet 357, 1225-1240.

Lazarevic, V., Margot, P., Soldo, B. \& Karamata, D. (1992). Sequencing and analysis of the Bacillus subtilis lytRABC divergon: a regulatory unit encompassing the structural genes of the $\mathrm{N}$-acetylmuramoyl-L-alanine amidase and its modifier. $J$ Gen Microbiol 138, 1949-1961.

Lazarevic, V., Mauël, C., Soldo, B., Freymond, P.-P., Margot, P. \& Karamata, D. (1995). Sequence analysis of the $308^{\circ}$ to $311^{\circ}$ segment of the Bacillus subtilis 168 chromosome, a region devoted to cell wall metabolism containing non-coding grey holes which reveal chromosomal rearrangements. Microbiology 141, 329-335.

Liu, W., Eder, S. \& Hulett, F. M. (1998). Analysis of Bacillus subtilis $\operatorname{tag} A B$ and tagDEF expression during phosphate starvation identifies a repressor role for PhoP-P. J Bacteriol 180, 753-758.

Marmur, J. (1961). A procedure for the isolation of deoxyribonucleic acid from microorganisms. J Mol Biol 3, 208-218.

Mauël, C., Young, M. \& Karamata, D. (1991). Genes concerned with synthesis of poly(glycerol phosphate), the essential teichoic acid in Bacillus subtilis strain 168, are organized in two divergent transcription units. J Gen Microbiol 137, 929-941.

Mauël, C., Young, M., Monsutti-Grecescu, A., Marriot, S. A. \& Karamata, D. (1994). Analysis of Bacillus subtilis tag gene expression using transcriptional fusions. Microbiology 140, 2279-2288. 
Mauël, C., Bauduret, A., Chervet, C., Beggah, S. \& Karamata, D. (1995). In Bacillus subtilis 168, teichoic acid of the cross-wall may be different from that of the cylinder: a hypothesis based on transcription analysis of tag genes. Microbiology 141, 2379-2389.

Pooley, H. M., Abellan, F.-X. \& Karamata, D. (1991). A conditionallethal mutant of Bacillus subtilis 168 with a thermosensitive glycerol-3-phosphate cytidylyltransferase, an enzyme specific for the synthesis of the major wall teichoic acid. J Gen Microbiol 137, 921-928.

Pooley, H. M., Abellan, F.-X. \& Karamata, D. (1992). CDPglycerol:poly(glycerophosphate) glycerophosphatetransferase, which is involved in the synthesis of the major wall teichoic acid in Bacillus subtilis 168, is encoded by tagF (rodC). J Bacteriol 174, 646-649.

Sambrook, J., Fritsch, E. F. \& Maniatis, T. (1989). Molecular Cloning: a Laboratory Manual, 2nd edn. Cold Spring Harbor, NY: Cold Spring Harbor Laboratory.
Soldo, B., Lazarevic, V., Pagni, M. \& Karamata, D. (1999). Teichuronic acid operon of Bacillus subtilis 168. Mol Microbiol 31, 795-805.

Vicente, M., Kushner, S. R., Garrido, T. \& Aldea, M. (1991). The role of the 'gear-box' in the transcription of essential genes. Mol Microbiol 5, 2085-2091.

Ward, J. B. (1981). Teichoic and teichuronic acids: biosynthesis, assembly and location. Microbiol Rev 45, 211-243.

Young, M., Mauël, C., Margot, P. \& Karamata, D. (1989). Pseudoallelic relationship between non-homologous genes concerned with biosynthesis of polyglycerol phosphate and polyribitol phosphate teichoic acids in Bacillus subtilis strains 168 and W23. Mol Microbiol 3, 1805-1812.

Received 3 August 2001; revised 8 October 2001; accepted 22 October 2001. 